\title{
Interactions between Egg-eating Predator and Prey: The Effect of the Functional Response and of Age Structure
}

\author{
F. VAN DEN Bosch ${ }^{1}$ AND O. DiekManN \\ Centre for Mathematics and Computer Science, Kruislaan 413, 1098 SJ \\ Amsterdam, The Netherlands
}

[Received 1 July 1985 and in revised form 13 January 1986]

\begin{abstract}
In this paper we analyse an age-structured predator-prey model in which predators eat only very young prey. The model can be formulated'as a system of three Volterra integral equations with an implicitly defined non-linearity. An interpretation of the implicit relation is given. The linearized stability of the steady states is investigated. It turns out that concentration of the predator on very young individuals is a stabilizing mechanism. Furthermore, it is seen that a compound parameter which is a measure for the efficiency of the predator has a major influence on the stability of the steady states. If the efficiency of the predator decreases the steady state can become unstable and oscillations will arise. Furthermore it is seen from the model that the destabilizing effect of a juvenile period is stronger when it concerns the predator than when it concerns the prey species. In an appendix it is shown that an egg-eating predator and an indiscriminately eating predator can coexist in a stable steady state while feeding on only one prey species.
\end{abstract}

\section{Introduction}

MANY authors have introduced predator-prey models which take account of realistic biological mechanisms. Murdoch \& Oaten (1975) present a good review of the insight which can be gained from such studies. But many models still suffer from obvious shortcomings, a rather important one being that all individuals of the same species are treated as being equal. Such models ignore the well-known fact that demographic indices such as fecundity and survival probability as well as properties related to the predation process such as vulnerability or aggressivity vary with the age, weight, or some other physiological characteristic of the individuals.

Some authors have introduced age-dependent demographic properties into predator-prey models (see for instance Cushing \& Saleem (1982), Hastings \& Wollkind (1982)).

Recently, age dependence in parameters describing the predation process has received some attention. From the biological literature it is known that many predators do not eat all ages of prey indiscriminately. There are, for instance, many well-documented examples where predators eat only the very young individuals or the eggs of the prey (Nielson, 1980; Le Cren et al., 1977; Dawson,

\footnotetext{
${ }^{1}$ Present address: Institute of Theoretical Biology, University of Leiden, Groenhovenstraat 5, 2311 BT Leiden, The Netherlands.
} 
1979; Brown \& Diamond, 1984). In order to translate this observation into a manageable model, Gurtin \& Levine (1979) introduced the extreme case in which predation only affects the rate of recruitment of the prey. In other words, the predation takes place instantaneously at prey-age zero. This type of interaction is now commonly referred to as an 'egg-eating predator-prey relation'. In continuations of this study many authors have given different forms to the factor with which the birth-rate is reduced to the actual recruitment rate (Gurtin \& Levine, 1979; Thompson et al., 1982; Coleman \& Frauenthal, 1983). Diekmann et al. (1985) criticize all of these. The mistake that produces wrong results is that, in the derivation of the birth-rate reduction factor, rates are treated as numbers. A correct derivation, incorporating a general functional response, is given in Diekmann et al. (1985) and subsequently it is used in a model for a cannibalistic species.

In this paper we use the same approach to construct a model for an egg-eating predator. We will combine some analytical and numerical work and reveal some robust properties of the model. Analytically the stability of the non-trivial steady state is studied for the most simple case and for a prey species with a reproductive capacity slightly larger than the critical one. Next we turn to a special case involving step functions for the age-specific birth rates and predation index. The stability boundaries in this case are calculated numerically.

\section{The model}

Consider age-structured prey and predator populations with age distributions given by, respectively, $n_{1}(t, a)$ and $n_{2}(t, \tau)$. (We shall, for the sake of clarity, systematically denote prey age by $a$ and predator age by $\tau$.) The evolution of these distributions is governed by the well-known McKendrick equation.

$$
\begin{aligned}
& \frac{\partial}{\partial t} n_{1}(t, a)+\frac{\partial}{\partial a} n_{1}(t, a)=-v(t, a) n_{1}(t, a), \\
& \frac{\partial}{\partial t} n_{2}(t, \tau)+\frac{\partial}{\partial \tau} n_{2}(t, \tau)=-\omega(t, \tau) n_{2}(t, \tau),
\end{aligned}
$$

with the boundary conditions

$$
\begin{aligned}
& n_{1}(t, 0)=b_{1}(t), \\
& n_{2}(t, 0)=b_{2}(t) .
\end{aligned}
$$

In order to define a complete model we have to specify the birth and death rates for both populations.

Let $B(a)$ be the expected number of young produced by a prey individual of age $a$ per unit of time. We assume that the birth rate of the prey is density-independent, so we take

$$
b_{1}(t)=\int_{0}^{\infty} B(a) n_{1}(t, a) \mathrm{d} a .
$$

The birth rate of the predator does depend on the amount of prey eaten and on 
the reproductive capacity of the predator. The two quantities of importance here are the attack rate of the predator on the prey and the conversion efficiency of prey, eaten by a predator, into new predators. We assume that prey age and predator age will influence each of these quantities independently. The attack rate is given by $\pi(\tau) A(a)$, where

$$
\begin{aligned}
& A(a)=\text { the prey-age-specific attack rate, } \\
& \pi(\tau)=\text { the predator-age-specific predation index. }
\end{aligned}
$$

These functions can be normalized in various ways. We will normalize such that $\pi(\tau) \in[0,1]$ and $\pi(\tau)=1$ for at least one $\tau$. The conversion factor is given by $\beta(\tau) \xi(a)$, where

$$
\begin{aligned}
& \zeta(a)=\text { the maximal number of new predators from a prey of age } a \\
& \beta(\tau)=\text { the predator age-specific reproduction index normalized such that } \\
& \\
& \beta(\tau) \in[0,1] \text { and } \beta(\tau)=1 \text { for at least one } \tau \text {. }
\end{aligned}
$$

The birth rate of the predator at a certain time $t$ depends linearly on the rate at which prey is eaten at that time. So

$$
b_{2}(t)=\int_{0}^{\infty} \pi(\tau) \beta(\tau) n_{2}(t, \tau) \mathrm{d} \tau \int_{0}^{\infty} \zeta(a) A(a) n_{1}(t, a) \mathrm{d} a \Phi\left(N_{1}(t)\right),
$$

where $\Phi\left(N_{1}(t)\right)$ is a correction factor which we will explain later on.

For the predator population we assume that the death rate only depends on the age of the predator and take:

$$
\omega(t, \tau)=\omega(\tau)
$$

The death rate of the prey consists of two terms both of which are prey-age-specific. The first is the rate of death $(\mu(a))$ due to causes other than predation. The second is the rate of death due to predation:

$$
v(t, a)=\mu(a)+A(a) N_{2}(t) \Phi\left(N_{1}(t)\right)
$$

with

$$
\begin{gathered}
N_{2}(t)=\int_{0}^{\infty} \pi(\tau) n_{2}(t, \tau) \mathrm{d} \tau, \\
N_{1}(t)=\int_{0}^{\infty} H(a) A(a) n_{1}(t, a) \mathrm{d} a,
\end{gathered}
$$

where:

$\mu(a)=$ the age-specific death-rate of the prey;

$\Phi\left(N_{1}(t)\right)=$ the correction factor for density-dependent effects; below we shall interpret $\Phi$ as the fraction of the time that a predator spends searching at prey density $n_{1}$;

$H(a)=$ the prey-age-specific handling time. 
We can interpret $N_{2}$ as the effective number of predators and $N_{1}$ as the effective number of potential victims (see below).

The functional response, i.e. the number of prey eaten per predator per unit of time, is now given by

$$
F\left(n_{1}(t, a)\right)=\int_{0}^{\infty} A(a) n_{1}(t, a) \mathrm{d} a \Phi\left(N_{1}(t)\right) .
$$

We will make the, biologically reasonable, assumption that $N_{1} \Phi\left(N_{1}\right)$ is increasing for $N_{1} \geqslant 0$ and that $\Phi\left(N_{1}\right)$ is decreasing for $N_{1} \geqslant 0$. Since $A N_{2} \Phi$ occurs as a product in equation (2.1) we can normalize $\Phi$ in various ways. We will normalize such that $\Phi(0)=1$ and $\lim _{N_{1} \rightarrow \infty} N_{1} \Phi\left(N_{1}\right)=1$. Here we assume that the limit exists and is finite. (As a side remark we mention that the Lotka-Volterra linear functional response corresponds to the choice $\Phi\left(N_{1}\right) \equiv 1$.)

For instance we can take:

$$
\Phi\left(N_{1}(t)\right)=\frac{1}{1+N_{1}(t)}
$$

then the functional response is the age-structured analogue of the Holling type II functional response. This can be seen from the following argument. The total time a predator spends handling prey $\left(T_{h}\right)$ equals the handling time per prey times the total number of catches. The total number of catches equals the attack rate multiplied by the population density and the total time spent searching $\left(T_{s}\right)$. In the age-structured model we get:

$$
\int_{0}^{\infty} H(a) A(a) n_{1}(t, a) \mathrm{d} a T_{s}=T_{h} .
$$

So in this context $N_{1}$ is the ratio of the time spent handling and the time spent searching and as such it is a convenient measure for the overall prey density. Holling $(1959,1966)$ argued that the total time available to the predator $T$ is the sum of the total handling time and the searching time $\left(T=T_{s}+T_{h}\right)$. Substitution in (2.4) gives:

$$
\frac{T_{s}}{T}=\frac{1}{1+\int_{0}^{\infty} H(a) A(a) n_{1}(t, a) \mathrm{d} a}=\frac{1}{1+N_{1}},
$$

which is exactly formula (2.3) for the correction factor $\Phi\left(N_{1}\right)$.

The general model is now complete. It allows for predation varying with the age of the prey and with the age of the predator in an independent manner. Next, we specialize to predation on the youngest individuals only. In the limiting case that we are going to study, the predation takes place instantaneously at $a=0$. The basic idea is to let the age interval in which the prey suffers from predation, as described by the support of $A(a)$, tend to zero while at the same time increasing the risk per unit of time of falling a victim to predation in such a way that the total risk, as described by $\theta=\int_{0}^{\infty} A(a) \mathrm{d} a$ remains strictly positive. The parameter $\theta$ can be considered as a vulnerability index.

We refer to Diekmann et al. (1985) for the technical details of the limit 
procedure. The limit model takes the form:

$$
\begin{gathered}
\frac{\partial n_{1}}{\partial t}+\frac{\partial n_{1}}{\partial a}=-\mu(a) n_{1}(t, a), \\
n_{1}(t, 0)=b_{1}(t) \exp \left[-\theta N_{2}(t) \Phi\left(N_{1}(t)\right)\right], \\
\frac{\partial n_{2}}{\partial t}+\frac{\partial n_{2}}{\partial \tau}=-\omega(\tau) n_{2}(t, \tau), \\
n_{2}(t, 0)=b_{2}(t)
\end{gathered}
$$

where

$$
\begin{gathered}
b_{1}(t)=\int_{0}^{\infty} B(a) n_{1}(t, a) \mathrm{d} a, \\
b_{2}(t)=\zeta H^{-1} N_{1} \Phi\left(N_{1}\right) \int_{0}^{\infty} \beta(\tau) \pi(\tau) n_{2}(t, \tau) \mathrm{d} \tau, \\
N_{2}(t)=\int_{0}^{\infty} \pi(\tau) n_{2}(t, \tau) \mathrm{d} \tau, \\
\frac{N_{1}(t) \Phi\left(N_{1}(t)\right)}{H}=\frac{b_{1}(t)}{N_{2}(t)}\left\{1-\exp \left[-\theta N_{2}(t) \Phi\left(N_{1}(t)\right)\right]\right\},
\end{gathered}
$$

with $\zeta=\zeta(0)$ and $H=H(0)$. For $N_{2}(t)=0$ the right-hand side of (2.5h) should be interpreted as $\theta b_{1}(t) \Phi\left(N_{1}(t)\right)$.

From $(2.5 \mathrm{~h})$ we see that the predation rate is determined by an implicit relation. The left-hand side is equal to the limit of the functional response (2.2). The right-hand side is, indeed, equal to the number of prey eaten per predator per unit of time. Hence $(2.5 \mathrm{~h})$ is a consistency condition. The assumptions on $N_{1} \Phi\left(N_{1}\right)$ and $\Phi\left(N_{1}\right)$ ensure that $N_{1}$ is uniquely determined as a function of $b_{1}$ and $N_{2}$ by this implicit relation.

System (2.5) will be the subject of our analysis in the next paragraph.

\section{Steady state and stability}

By integration along characteristics we can reduce the limit-model to a system of three Volterra integral equations and one scalar equation:

$$
\left.\begin{array}{l}
b_{1}(t)=\gamma \int_{0}^{\infty} g(a) b_{1}(t-a) \exp \left[-\theta N_{2}(t-a) \Phi\left(N_{1}(t-a)\right)\right] \mathrm{d} a, \\
b_{2}(t)=\zeta H^{-1} \delta N_{1} \Phi\left(N_{1}\right) \int_{0}^{\infty} f(\tau) b_{2}(t-\tau) \mathrm{d} \tau, \\
N_{2}(t)=\alpha \int_{0}^{\infty} h(\tau) b_{2}(t-\tau) \mathrm{d} \tau, \\
\frac{N_{1}(t) \Phi\left(N_{1}(t)\right)}{H}=\frac{b_{1}(t)}{N_{2}(t)}\left\{1-\exp \left[-\theta N_{2}(t) \Phi\left(N_{1}(t)\right)\right]\right\},
\end{array}\right\}
$$


where

$$
\begin{aligned}
\gamma g(a) & =B(a) \mathrm{e}^{-\int_{0}^{a} \mu(\sigma) \mathrm{d} \sigma}, \\
\delta f(\tau) & =\pi(\tau) \beta(\tau) \mathrm{e}^{-\int_{0}^{\tau} \omega(\sigma) \mathrm{d} \sigma}, \\
\alpha h(\tau) & =\pi(\tau) \mathrm{e}^{-\int_{0}^{\tau} \omega(\sigma) \mathrm{d} \sigma},
\end{aligned}
$$

and $\gamma, \delta$, and $\alpha$ are chosen such that $g(a), f(\tau)$, and $h(\tau)$ have integral one. Consequently:

$\gamma=$ the expected number of offspring produced by one newly-recruited prey individual during the course of its future life.

$\delta=$ the expected time a predator spends searching and eating during the whole course of its reproductive life.

$\alpha=$ the expected time a predator spends searching and handling during its entire life.

We will study the existence and local stability of the steady states of this system. For a treatment of the theory of linearized stability of Volterra convolution integral equations we refer to Diekmann \& van Gils (1984).

Besides the trivial steady state

$$
\left(\hat{b}_{1}, \hat{b}_{2}, \hat{N}_{2}, \hat{N}_{1}\right)=(0,0,0,0)
$$

we also find a non-trivial steady state from:

$$
\left.\begin{array}{l}
\hat{N}_{1} \Phi\left(\hat{N}_{1}\right)=\frac{H}{\zeta \delta}, \\
\hat{b}_{1}=\hat{N}_{1} \frac{1}{\theta H} \frac{\gamma \ln \gamma}{\gamma-1}, \\
\hat{b}_{2}=\frac{1}{\alpha} \frac{\ln \gamma}{\theta \Phi\left(\hat{N}_{1}\right)}, \\
\hat{N}_{2}=\frac{\ln \gamma}{\theta \Phi\left(\hat{N}_{1}\right)} .
\end{array}\right\}
$$

The assumptions on $\Phi$ ensure that the first equation has a unique solution (which does not depend on $\gamma$ !) provided that $\zeta \delta / H>1$. From the interpretation of $\delta$ and $H$ we see that $\delta / H$ is the maximal number of victims a predator can make during the whole course of its life. Multiplying this quantity with the conversion factor $\zeta$ gives the maximal number of offspring a predator can have. When $\zeta \delta / H<1$ the predator population becomes extinct no matter how large the prey population is. We will call $\zeta \delta / H$ the efficiency factor of the predator.

In the special case $\Phi\left(N_{1}\right)=1 /\left(1+N_{1}\right)$ we find explicitly

$$
\hat{N}_{1}=\frac{1}{\frac{\zeta \delta}{H}-1}
$$




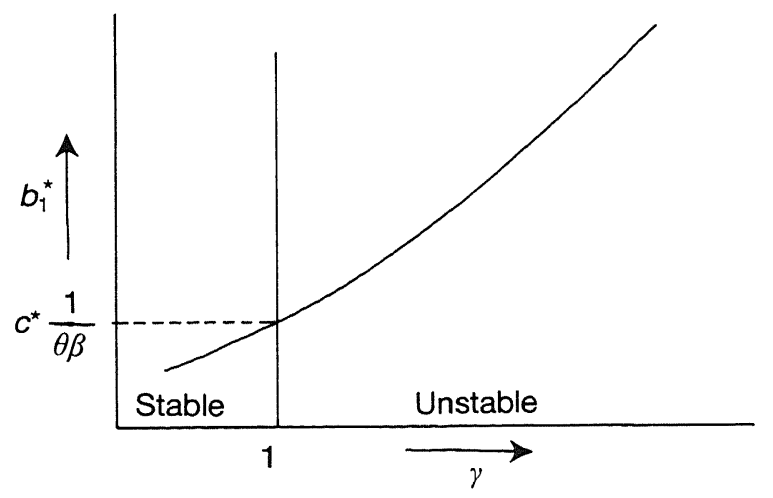

FIG. 1. Bifurcation diagram.

For $\gamma=1$ there are also the steady states

$$
\left.\begin{array}{l}
N_{1}^{*}=H \theta b_{1}^{*}, \\
b_{1}^{*} \in \mathbb{R}, \text { arbitrary }, \\
b_{2}^{*}=0, \\
N_{2}^{*}=0 .
\end{array}\right\}
$$

The bifurcation diagram is depicted in Fig. 1. Note the vertical bifurcation at $\gamma=1$.

Linearization of (3.1) about the trivial steady state yields the characteristic equation

where

$$
\gamma \bar{g}(\lambda)-1=0
$$

$$
\bar{g}(\lambda)=\int_{0}^{\infty} \mathrm{e}^{-\lambda a} g(a) \mathrm{d} a
$$

and we can conclude that the trivial steady state is stable if $\gamma<1$ and unstable if $\gamma>1$ in accordance with the biological interpretation of $\gamma$.

Linearization about the non-trivial steady state (3.2) yields, after some calculations, the characteristic equation

where

$$
\begin{aligned}
& \bar{g}(\lambda)\{\bar{h}(\lambda)(1-q) k(\gamma)+(\bar{f}(\lambda)-1)[q k(\gamma)-1]\} \\
& -\bar{h}(\lambda)(1-q) m(\gamma)-[\bar{f}(\lambda)-1][q m(\gamma)+1]=0
\end{aligned}
$$

$$
\begin{gathered}
k(\gamma)=\frac{\ln \gamma}{\gamma-1}-1+\ln \gamma, \\
m(\gamma)=\frac{\ln \gamma}{\gamma-1}-1=k(\gamma)-\ln \gamma,
\end{gathered}
$$

and

$$
q=-\hat{N}_{1} \frac{\Phi^{\prime}\left(\hat{N}_{1}\right)}{\Phi\left(\hat{N}_{1}\right)}
$$


The assumptions about $\Phi\left(N_{1}\right)$ imply that the parameter $q$ necessarily is confined to the interval $(0,1)$.

For our special case $\Phi\left(N_{1}\right)=1 /\left(1+N_{1}\right)$ we find

$$
q=\frac{\zeta \delta}{H}
$$

which is the efficiency factor of the predator as we have defined earlier. (As an aside note that $\Phi\left(N_{1}\right) \equiv 1$ yields $q=0$.)

Equation (3.4) is rather unwieldy. We will study some special cases in order to obtain some insight.

\subsection{The Most Simple Case}

Suppose that, except for the fact that predation takes place at prey age $a=0$, all indices are age-independent. So, in particular,

$$
\begin{gathered}
g(a)=\mu \mathrm{e}^{-\mu a}, \\
h(\tau)=f(\tau)=\omega \mathrm{e}^{-\omega \tau} .
\end{gathered}
$$

Note that this implies that both $\pi$ and $\beta$ are identically one. Straightforward manipulations applied to either (2.5) or (3.1) lead to the system of ordinary differential equations

$$
\left.\begin{array}{rl}
\frac{\mathrm{d} n}{\mathrm{~d} t} & =\mu \gamma n \exp \left[-\theta N_{2} \Phi\left(N_{1}\right)\right]-\mu n \\
\frac{\mathrm{d} N_{2}}{\mathrm{~d} t} & =\zeta H^{-1} N_{1} \Phi\left(N_{1}\right) N_{2}-\omega N_{2}
\end{array}\right\}
$$

with $N_{1}$ as a function of $n$ and $N_{2}$ defined by

and

$$
\frac{N_{1} \Phi\left(N_{1}\right)}{H}=\mu \gamma \frac{n}{N_{2}}\left\{1-\exp \left[-\theta N_{2} \Phi\left(N_{1}\right)\right]\right\}
$$

$$
\begin{aligned}
& n(t)=\int_{0}^{\infty} n_{1}(t, a) \mathrm{d} a=\text { total prey population, } \\
& N_{2}(t)=\int_{0}^{\infty} n_{2}(t, \tau) \mathrm{d} \tau=\text { total predator population. }
\end{aligned}
$$

Of course one can also write down (3.5) directly from the assumptions and the interpretation.

The characteristic equation (3.4) now becomes

$$
e \lambda^{2}+b \lambda+c=0
$$

where

$$
\begin{aligned}
e & =1+q m(\gamma) \\
b & =-q \mu[k(\gamma)-m(\gamma)]+\omega m(\gamma)(q-1), \\
c & =\omega \mu[k(\gamma)-m(\gamma)](1-q) .
\end{aligned}
$$


Since $e \geqslant 0$ and $c \geqslant 0$ for $\gamma \geqslant 1$ and $q \in(0,1)$ we conclude that the steady state is stable if $b>0$ and unstable if $b<0$. The criticality condition $b=0$ defines the stability boundary in parameter space. Expressing $q$ as a function of the other parameters we find

$$
q\left(\gamma, \frac{\mu}{\omega}\right)=\frac{-m(\gamma)}{\frac{\mu}{\omega} \ln \gamma-m(\gamma)}=\frac{\ln \gamma-\gamma+1}{\ln \gamma-\gamma+1-\frac{\mu}{\omega}(\gamma-1) \ln \gamma} .
$$

Some important properties are:

$$
\lim _{\gamma \downarrow 1} q\left(\gamma, \frac{\mu}{\omega}\right)=\frac{1}{2 \frac{\mu}{\omega}+1},
$$

(ii) $\quad \lim _{\gamma \rightarrow \infty} q\left(\gamma, \frac{\mu}{\omega}\right)=0$,

(iii)

$$
\frac{\partial}{\partial \gamma} q\left(\gamma, \frac{\mu}{\omega}\right)=\frac{\mu}{\omega} \frac{(\ln \gamma)^{2}-(\gamma-1)^{2} / \gamma}{\left(\ln \gamma-\gamma+1-\frac{\mu}{\omega}(\gamma-1) \ln \gamma\right)^{2}}<0 \text { for } \gamma>1 \text {, }
$$

(iv)

$$
\frac{\partial}{\partial \frac{\mu}{\omega}} q\left(\gamma, \frac{\mu}{\omega}\right)<0 \text { for } \gamma>1
$$

In Fig. 2 we display $q$ as a function of $\gamma$ for various values of $\mu / \omega$. We see that the form of the stability boundary in the $(\gamma, q)$-plane is not influenced by the value of $\mu / \omega$.

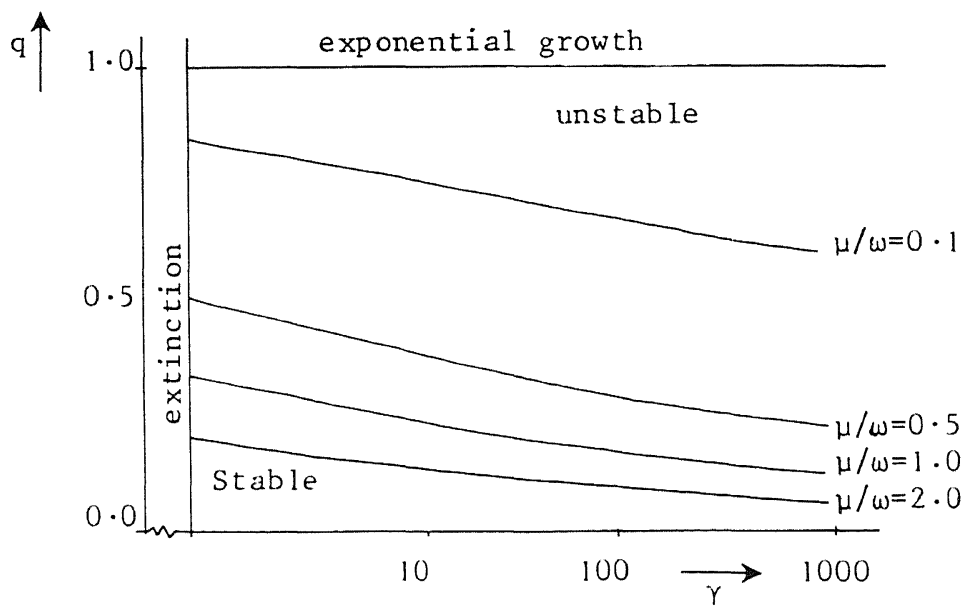

FIG. 2. The stability boundary in the $(\gamma, q)$-plane of 'the most simple case' for various values of $\mu / \omega$. 
In the case of a linear functional response $(q=0)$ the steady state is always stable. In fact the corresponding system

$$
\left.\begin{array}{rl}
\frac{\mathrm{d} n}{\mathrm{~d} t} & =\mu n\left(\gamma \mathrm{e}^{-N_{2}}-1\right) \\
\frac{\mathrm{d} N_{2}}{\mathrm{~d} t} & =\zeta n\left(1-\mathrm{e}^{-\theta N_{2}}\right)-\omega N_{2}
\end{array}\right\}
$$

has a globally asymptotically stable steady state, as can be deduced from Bendixson's criterion after the change of variables $n=\mathrm{e}^{m}, N_{2}=\mathrm{e}^{R}$.

In Appendix III we modify the system (3.7) to account for the presence of yet another predator species which eats all prey indiscriminately and make a remark about the competitive exclusion principle.

\section{(ii) Prey with Small Reproductive Capacity}

For $y=1$ the characteristic equation (3.4) has two roots equal to zero. The stability of the non-trivial steady state (3.2) for $\gamma$ slightly larger than one depends on the sign of the real part of these two critical roots. The procedure to assess the stability properties of the steady state in such a situation is explained in Appendix 1. With this procedure we find that the steady state is stable if and only if

$$
(q-1) p+r<0
$$

where

with

$$
\begin{gathered}
p=\tilde{f} \dot{g}+\tilde{g} \tilde{f}-2 \tilde{g} \tilde{h} \tilde{f}+3 \tilde{g} \tilde{f}^{2}, \\
p=2 \tilde{g} \tilde{f}^{2},
\end{gathered}
$$

$$
\begin{aligned}
& g=\int_{0}^{\infty} a g(a) \mathrm{d} a=\text { the mean of } g(a), \\
& g=\int_{0}^{\infty}(a-\bar{g})^{2} g(a) \mathrm{d} a=\text { the variance of } g(a),
\end{aligned}
$$

and similar definitions for $f$ and $h$.

From (3.8) we see that a reduction of the efficiency of the predator (i.e. an increase of $q$ ) can cause a stable steady state to become unstable. In the limit $q \uparrow 1$ the steady state is always unstable.

If we take $\tilde{h}=\hat{f}$, for instance by putting $\beta(\tau) \equiv 1,(3.8)$ can be written as:

$$
q<\frac{-2 \tilde{g} \tilde{f}^{2}}{f g+f \tilde{g}+\tilde{g} \tilde{f}^{2}}+1 .
$$

From (3.9) we see that an increase of the mean age of reproduction of any of the species can destabilize a stable steady state. If we let $\tilde{g} \rightarrow \infty$ for fixed $\tilde{f}, \tilde{\tilde{f}}$, and $g$ the condition becomes

$$
q<1-\frac{2 f^{2}}{f+f^{2}}
$$


which still can be satisfied for some positive values of $q$ provided $\tilde{\tilde{f}}>\tilde{f}^{2}$. However, if $\tilde{f} \rightarrow \infty$ for fixed $\tilde{g}, \tilde{\tilde{g}}$, and $\tilde{\tilde{f}}$ we find

$$
q<-1 \text {, }
$$

which clearly is impossible for positive $q$. So in this sense the mean age of reproduction of the predator can have a stronger destablizing influence than the mean age of reproduction of the prey.

\section{(iii) Delayed Reproduction and Predation}

Next we take the death rate to be age-independent, and the age-specific birth rates and the predation index to be step functions. So,

$$
\begin{aligned}
& g(a)=\mathrm{H}\left(a-T_{1}\right) \mu \mathrm{e}^{-\mu\left(a-T_{1}\right)}, \\
& f(\tau)=\mathrm{H}\left(\tau-T_{3}\right) \omega \mathrm{e}^{-\omega\left(\tau-T_{3}\right)}, \\
& h(\tau)=\mathrm{H}\left(\tau-T_{2}\right) \omega \mathrm{e}^{-\omega\left(\tau-T_{2}\right)} .
\end{aligned}
$$

Here, $\mathrm{H}(\bullet)$ the Heaviside step function, $T_{1}$ and $T_{3}$ are the juvenile period of the prey and predator respectively, and $T_{2}$ is the pre-predatory period. So $T_{2} \leqslant T_{3}$. The stability boundary is determined numerically with a procedure described in Appendix II.

From Figs 3, 4, and 5 we see that the form of the stability boundary is not much influenced by variation of the delays in any of the species. The figures shown here are representative for most parameter values we investigated. The general picture is that the stability domain becomes smaller when $\mu / \omega$ or any of the delays is increased. A comparison of Figs 3 and 4 gives the impression that the stability domain is reduced more when the juvenile period of the predator is increased than when the juvenile period of the prey is increased.

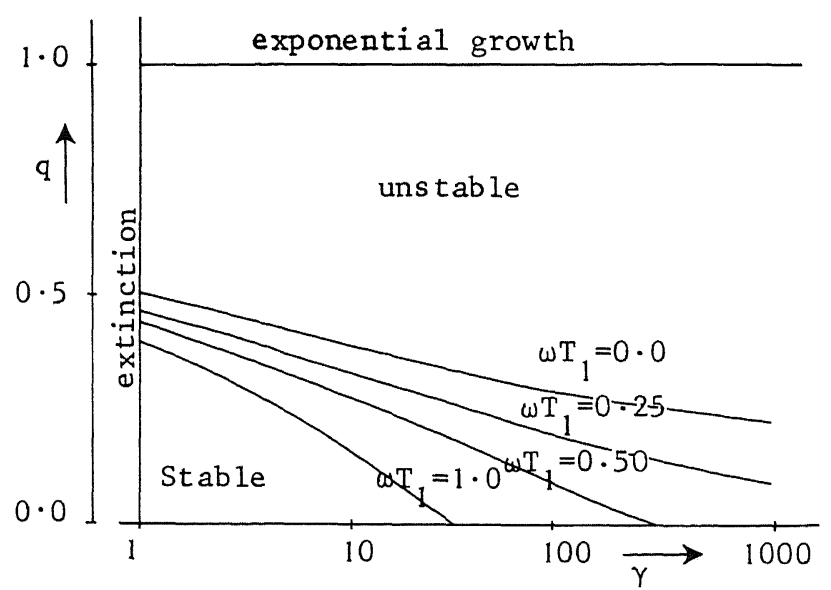

FIG. 3. The stability boundary for various values of $\omega T_{1}$ when $\omega T_{2}=\omega T_{3}=0.0$ and $\mu / \omega=0.5$. 


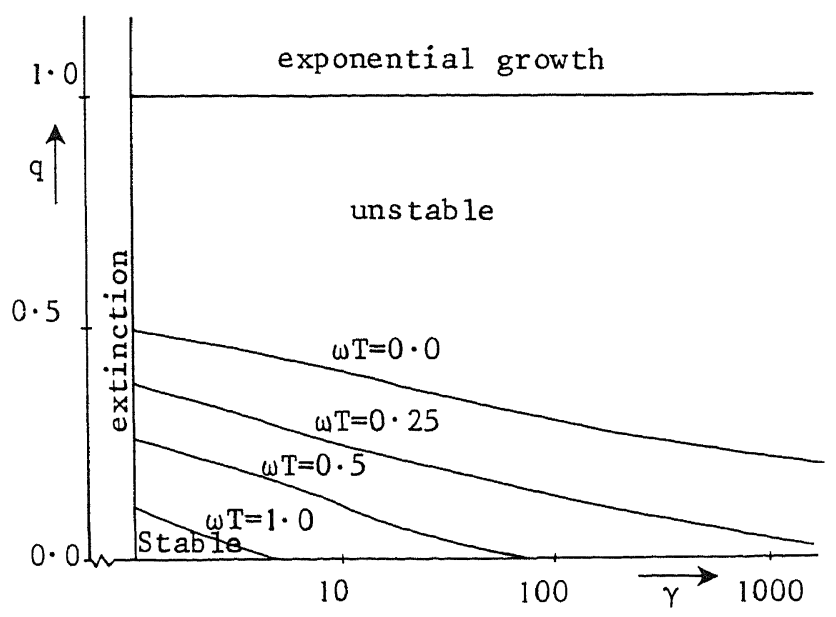

FIG. 4. The stability boundary for various values of $\omega T_{2}=\omega T_{3}=\omega T$ when $\omega T_{1}=0.0$ and $\mu / \omega=0.5$. For $\omega T>\sqrt{ } 2$ all non-trivial steady states are unstable.

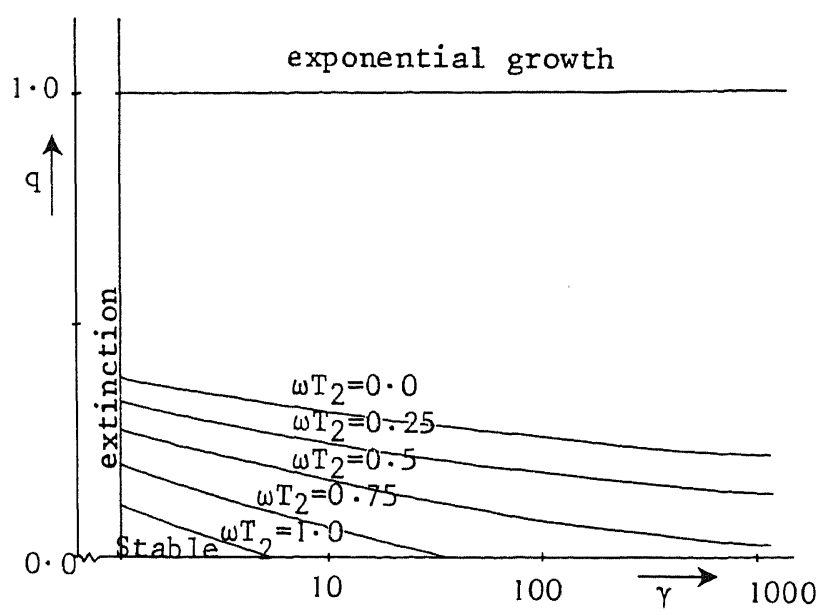

FIG. 5. The stability boundary for various values of $\omega T_{2}$ when $\omega T_{1}=0.0, \omega T_{3}=1 \cdot 0$, and $\mu / \omega=0.5$.

When $T_{2} \neq T_{3}$ restabilization can occur when $T_{3}$ is increased when $\mu / \omega$ and $\omega T_{3}$ are large. This restabilization occurs in a small interval of $q$ values. Although this phenomenon is rather intriguing, we consider it, in this model, as biologically irrelevant.

In Fig. 6 we illustrate the destabilizing effect of the juvenile periods in more detail. This figure is representative for all parameter values investigated. From Figs 3,4 , and 6 we see that the juvenile period of the predator is more critical to the stability of the steady state than the juvenile period of the prey. We will return to this point in the discussion. 


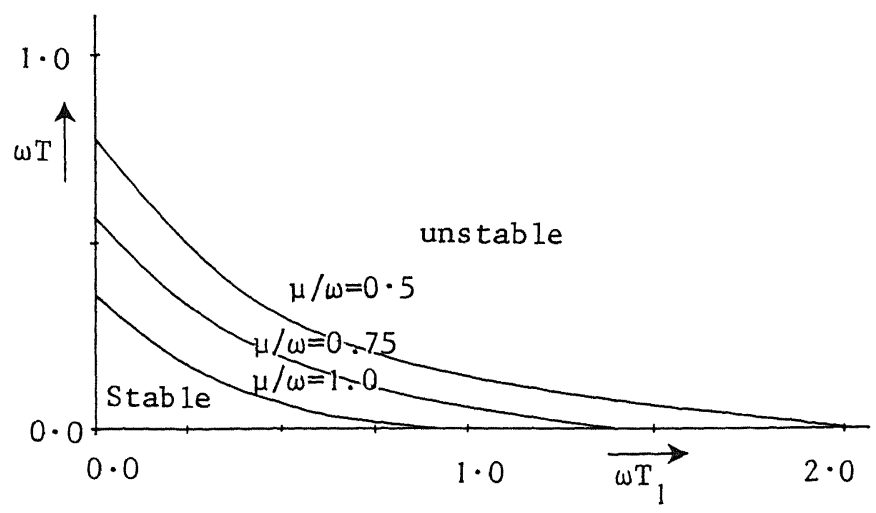

FIG. 6. The stability boundary in the $\left(\omega T_{1}, \omega T\right)$-plane, where $\omega T=\omega T_{2}=\omega T_{3}$, for $q=0 \cdot 0, \gamma=10 \cdot 0$, and various values of $\mu / \omega$.

The period $P$ of the periodic solution that arises at the stability boundary seems to depend mainly on $\gamma$ and not so much on other parameters. We found that $P$ decreases with increasing $\gamma$. We found $P \approx 10$ for $\gamma=10, P \approx 6$ for $\gamma=100$, and $P \approx 4$ for $\gamma=1000$. The time unit in which the period is expressed is 'the mean longevity of the predator' $(1 / \omega)$.

\section{Discussion}

The model we have analysed in this paper is based on some assumptions that will not be met in the real world. Density-dependent effects other than predation are not incorporated in the model. For instance, self-regulation of the prey and/or the predator can be important when the population density is high. These points should be given some attention before we can draw general conclusions about the dynamical consequences of relations between egg-eating predators and their prey, but some conclusions can already be drawn from the results of Section 3.

The well-known Lotka-Volterra model, where the prey is eaten indiscriminately, can be compared with our 'most simple case' with a linear functional response. The Lotka-Volterra model has a neutrally stable steady state. The egg-eating-predator model has a globally stable steady state. So, concentration of the predator on very young prey can be considered as a stabilizing mechanism. With model formulations that are disputable (see Section 1) Gurtin \& Levine (1979) concluded that egg eating is destabilizing while Thompson et al. (1982) concluded that it is stabilizing.

The functional response, expressed here through the efficiency factor $q$, is seen to act as a destabilizing mechanism. It has a major influence on the stability of the non-trivial steady state. When the predator is very inefficient the steady state is always unstable. Coleman \& Frauenthal (1983) also investigated an egg-eatingpredator model where the predator has a non-linear functional response. They arrived at the opposite conclusion, but, as argued in Section 1, their model formulation is disputable. 
Delays in reproduction and predation are also seen to be destabilizing. A striking feature of our results is that the range of juvenile periods for which the steady state is stable is larger in the prey than in the predator species. Hastings (1984) analysed a model where adult prey individuals are eaten by adult predators. In that model the juvenile period of the prey proved to be more critical than the juvenile period of the predator. Furthermore, he found that when the juvenile period of the prey is smaller than or equal to that of the predator, the steady state is always unstable. These conclusions differ from the ones derived in the present paper and might point to a qualitative difference between predation on adults and predation on very young individuals.

In conclusion we can say that concentration of the predator on very young individuals is stabilizing, a non-linear functional response and delays in reproduction and predation are destabilizing. The conflicting effects of these mechanisms determine the precise location of the stability boundary.

\section{Acknowledgement}

We are grateful to R. M. Nisbet for stimulating discussions and advice, and for drawing our attention to the paper by Haigh \& Maynard Smith. We wish to thank two anonymous referees who provided valuable criticism.

\section{REFERENCES}

Brown, A. F., \& DiAmond, M. 1984 The consumption of rainbow trout (Salmo gairdneri Richardson) eggs by macroinvertebrates in the field. Freshwater Biol. 14, 211-215.

Coleman, C. S., \& Frauenthal, J. C. 1983 Satiable egg eating predators, Math. Biosc. $63,99-119$.

Cushing, J. M., \& Saleem, M. 1982 A predator prey model with age structure. J. Math. Biol. 14, 231-250.

DAwson, P. S. 1979 Evolutionary changes in egg-eating behaviour of flour beetles in mixed-species populations. Evolution 33, 585-594.

Diekmann, O., Nisbet, R. M., Gurney, W. S. C., \& v.D. Bosch, F. 1985 Simple mathematical models for cannibalism: A critique and a new approach. Math. Biosc. 78, 21-46.

DiekmanN, O., \& VAN Gils, S. A. 1984 Invariant manifolds for Volterra-integral equations of convolution type. J. diff. Equ. 54, 139-180.

Gurtin, M. E., \& Levine, D. S. 1979 On predator-prey interactions with predation dependent on age of prey. Math. Biosc. 47, 207-219.

HAIGH, J., \& MAYNARD SMITH, J. 1972 Can there be more predators than prey? Theor. Pop. Biol. 3, 290-299.

Hastings, A. 1984 Delays in recruitment at different trophic levels: Effects on stability. $\boldsymbol{J}$. Math. Biol. 21, 35-44.

Hastings, A., \& Wollkind, D. 1982 Age structure in predator-prey systems, I: A general model and a specific example. Theor. Pop. Biol. 21, 44-57.

Holling, C. S. 1959 Some characteristics of simple types of predation and parasitism. Can. Entomol. 91, 385-398.

Holling, C. S. 1966 The functional response of invertebrate predators to prey density. Mem. Ent. Soc. Canada 48.

Le Cren, E. D., Kipling, C., \& McCormack, J. C. 1977 A study of the number, biomass and year-class strengths of perch (Perca fhiviatilis L.) in Windermere from 1941 to 1966. J. Amin. Ecol. 46, 281-307. 
Murdoch, W. W., \& OAten, A. 1975 Predation and population stability. Adv. Ecol. Res. 9, 1-131.

Nielson, L. A. 1980 Effect of Walleye (Stizostedion vitreum vitreum) predation on juvenile mortality and recruitment of yellow perch (Perca flavescens) in Oneida lake, New York. Can. J. Fish. Aqua. Sc. 37, 11-19.

Thompson, R. W., Di Biasio, D., \& Mendes, C. 1982 Predator-prey interactions: Egg-eating predators. Math. Biosc. 60, 109-120.

Waltman, P. 1983 Competition models in population biology. SIAM Regional Conference Series in Applied Math. 45.

\section{Appendix I}

To assess the stability of the non-trivial steady state for $\gamma$ slightly larger than one put $\gamma=1+\varepsilon$ and consider the characteristic equation as a function $F$ of $\lambda$ and $\varepsilon$. The expansion of $F(\lambda, \varepsilon)$ about $\lambda=\varepsilon=0$ is given by

$$
F(\lambda, \varepsilon)=A \varepsilon+B \lambda^{2}+C \varepsilon \lambda+D \lambda^{3}+\cdots
$$

since $(\partial F / \partial \lambda)_{\lambda=0 ; \varepsilon=0}=0$. Assume that $\lambda$ can be expanded in powers of $\sqrt{ } \varepsilon$, i.e.

$$
\lambda=c_{1} \sqrt{ } \varepsilon+c_{2} \varepsilon+\cdots
$$

Substituting this into the expansion for $F$ we find after equalizing like powers of $\vee \varepsilon$ :

$$
\begin{aligned}
& c_{1}= \pm \sqrt{ }\left(-\frac{A}{B}\right) \\
& c_{2}=\frac{A D-B C}{2 B^{2}} .
\end{aligned}
$$

We conclude that:

(i) the steady state is stable if

$$
\frac{A}{B}>0 \text { and } A D-B C<0 ;
$$

(ii) the steady state is unstable if

$$
\frac{A}{B}<0 \text { or } \frac{A}{B}>0 \text { and } A D-B C>0 .
$$

\section{Appendix II}

When studying characteristic equations like (3.4) we want to divide the space of parameters into two complementary regions: (i) the stability region where all roots lie in the left half plane and, as a consequence, the steady state is stable and (ii) the instability region where at least one root lies in the right half plane and, as a consequence, the steady state is unstable. The so-called stability boundary between these two regions is characterized by the fact that at least one root lies 
exactly on the imaginary axis. In this appendix we show how the stability boundary can be computed in an easy and systematic way if (i) we concentrate on two parameters, while keeping the other fixed and (ii) the characteristic equation is linear in one of these two parameters.

So, we want to find solutions of an equation of the form $F(\lambda, \alpha, \beta)=0$ where $\lambda$ is purely imaginary, the 'parameters' $\alpha$ and $\beta$ are real and $F$ is linear in $\alpha$. The equation can be written as:

or

$$
F(\lambda, \alpha, \beta)=F_{1}(\lambda, \beta)-\alpha F_{2}(\lambda, \beta)=0
$$

$$
\alpha=\frac{F_{1}(\lambda, \beta)}{F_{2}(\lambda, \beta)}=\frac{\operatorname{Re} F_{1}+\mathrm{i} \operatorname{Im} F_{1}}{\operatorname{Re} F_{2}+\mathrm{i} \operatorname{Im} F_{2}}
$$

Since $\alpha$ is real we necessarily should have

$$
-\operatorname{Re} F_{1} \operatorname{Im} F_{2}+\operatorname{Re} F_{2} \operatorname{Im} F_{1}=0 .
$$

This is an equation in two variables $\lambda$ and $\beta$. For fixed $\beta$ successive roots $\lambda$ can be found with standard numerical procedures. Subsequently $\alpha$ can be found from

if $\operatorname{Re} F_{2} \neq 0$ and

$$
\alpha=\frac{\operatorname{Re} F_{1}}{\operatorname{Re} F_{2}}
$$

$$
\alpha=\frac{\operatorname{Im} F_{1}}{\operatorname{Im} F_{2}}
$$

otherwise. Thus we construct curves in the $(\alpha, \beta)$-plane on which the equation $F=0$ has a purely imaginary root. The 'outer' one is the true stability boundary. The clue to this method is the first step in which the equation is 'solved' for $\alpha$. When studying (3.4) with $f, g$, and $h$ given by (3.10) we first gave $q$ the rôle of $\alpha$ and $\gamma$ the rôle of $\beta$. But when making Fig. 5 we used a preparatory log transformation to make the equation linear in $\omega T$ and then gave $\omega T$ the rôle of $\alpha$ and $\omega T_{1}$ the rôle of $\beta$.

\section{Appendix III}

Assuming linear functional responses, we may describe (after scaling all variables), the interaction of one prey $(n)$, one egg-eating predator $\left(N_{2}\right)$ and one indiscriminately eating predator $(R)$ by the system

$$
\begin{aligned}
\frac{\mathrm{d} n}{\mathrm{~d} t} & =n\left(\gamma \mathrm{e}^{-N_{2}}-1-R\right), \\
\frac{\mathrm{d} N_{2}}{\mathrm{~d} t} & =N_{2}\left(n f\left(N_{2}\right)-\omega\right), \\
\frac{\mathrm{d} R}{\mathrm{~d} t} & =R(\varepsilon n-\zeta),
\end{aligned}
$$

where $f\left(N_{2}\right)=\left(1-\mathrm{e}^{-N_{2}}\right) / N_{2}$ (so $f$ is monotone decreasing) and $\gamma, \omega$, $\varepsilon$, and $\zeta$ are 
positive parameters. In the invariant $n-R$ plane we have Lotka-Volterra dynamics: periodic orbits around the equilibrium $\tilde{n}=\zeta / \varepsilon, \tilde{R}=\gamma-1$. In the invariant $n-N_{2}$ plane there is a globally stable equilibrium $\bar{N}_{2}=\ln \gamma, \bar{n}=\omega[\gamma /$ $(\gamma-1)] \ln \gamma$. The coexistence equilibrium

$$
n^{*}=\zeta / \varepsilon, \quad N_{2}^{*}=f^{-1}\left(\frac{\varepsilon v}{\zeta}\right), \quad R^{*}=\gamma \mathrm{e}^{-N_{2}^{*}-1}
$$

is biologically relevant (i.e., lies in the positive octant) provided that

$$
\frac{\gamma-1}{\gamma \ln \gamma}<\frac{\varepsilon v}{\zeta}<1 .
$$

Under the condition $(*)$ both the $(\tilde{n}, 0, \tilde{R})$ and the $\left(\tilde{n}, \bar{N}_{2}, 0\right)$ equilibrium are unstable with respect to the three-dimensional system. Some further calculations show that the $*$ equilibrium cannot change its stability type while moving (as parameters are varied) from the $(\tilde{n}, 0, \tilde{R})$ equilibrium to the $\left(\bar{n}, \bar{N}_{2}, 0\right)$ equilibrium or vice versa and, moreover, that it is locally asymptotically stable near the bifurcation points. So we conclude that both predators can coexist in a stable steady state if (*) is satisfied, although there is only one food source. They manage to do so by specializing on different stages of the prey. This point was put forward by Haigh \& Maynard Smith (1972) in the context of a slightly more complicated model.

From a mathematical point of view it seems interesting to add saturating functional responses and carrying capacities, and to study, in the spirit of Waltman (1983), the movement of limit cycles from one invariant plane to another as parameters are varied. 